\title{
Comparing minimally supervised home-based and closely supervised gym-based exercise programs in weight reduction and insulin resistance after bariatric surgery: $A$ randomized clinical trial
}

\author{
Sara Kaviani ${ }^{1}$, Haleh Dadgostar ${ }^{2 *}$, Ali Mazaherinezhad ${ }^{2}$, Hanie Adib ${ }^{3}$, Masoud Solaymani-Dodaran ${ }^{4}$ \\ Fahimeh Soheilipour ${ }^{3}$, Mahdi Hakiminezhad ${ }^{5}$
}

Received: 5 May 2016

Published: 22 June 2017

\begin{abstract}
Background: Effectiveness of various exercise protocols in weight reduction after bariatric surgery has not been sufficiently explored in the literature. Thus, in the present study, we aimed at comparing the effect of minimally supervised home-based and closely supervised gym-based exercise programs on weight reduction and insulin resistance after bariatric surgery.

Methods: Females undergoing gastric bypass surgery were invited to participate in an exercise program and were randomly allocated into 2 groups using a random number generator in Excel. They were either offered a minimally supervised home-based (MSHB) or closely supervised gym-based (CSGB) exercise program. The CSGB protocol constitutes 2 weekly training sessions under ACSM guidelines. In the MSHB protocol, the participants received a notebook containing a list of recommended aerobic and resistance exercises, a log to record their activity, and a schedule of follow-up phone calls and clinic visits. Both groups received a pedometer. We measured their weight, BMI, lipid profile, FBS, and insulin level at baseline and at 20 weeks after the exercises, the results of which were compared using $t$ test or Mann-Whitney $U$ test at the end of the study. All the processes were observed by 1 senior resident in sport medicine.

Results: A total of 80 patients were recruited who were all able to complete our study (MSHB $=38$ and $\mathrm{CSGB}=42$ ). The baseline comparison revealed that the 2 groups were similar. The mean change (reduction) in BMI was slightly better in CSGB $(8.6195 \% \mathrm{CI}$ 7.76-9.45) compared with the MSHB (5.18 95\% CI 3.91-6.46); $p<0.01$. However, the 2 groups did not have a statistically significant difference in the amount of change in the other factors including FBS and Homa.ir.

Conclusion: As we expected a non-inferiority result, our results showed that both MSHB and CSGB exercise methods are somewhat equally effective in improving lipid profile and insulin resistance in the 2 groups, but a slightly better effect on BMI was observed in CSGB group. With considerably lower costs of minimally supervised home- based exercise programs, both methods should be considered when there is lack of adequate funding.
\end{abstract}

Keywords: Postoperative Exercise; Insulin Resistance; Bariatric Surgery; Morbid Obesity

Copyright $@$ Iran University of Medical Sciences

Cite this article as: Kaviani S, Dadgostar H, Mazaherinezhad A, Adib H, Solaymani-Dodaran M, Soheilipour F, Hakiminezhad M. Comparing minimally supervised home-based and closely supervised gym-based exercise programs on weight reduction and insulin resistance after bariatric surgery: A randomized clinical trial. Med J Islam Repub Iran. 2017 (22 June);31:34. https://doi.org/10.14196/mjiri.31.34

\section{Introduction}

Being overweight and obese is one of the health problems in many developed countries all around the world. Currently, more than 2 billion people, approximately $35 \%$ of the global population, are either overweight or obese. The prevalence of severe obesity continues to rise and it seems that half of the world population will be overweight

Corresponding author: Dr Haleh Dadgostar, hldadgostar@gmail.com

\footnotetext{
1. Sports Medicine department, Rasoul e Akram Hospital, Iran University of Medical Sciences, Tehran, Iran.

2. Minimally Invasive Surgery Research Center, Iran University of Medical Sciences, Tehran, Iran, \& Sports Medicine Department, Rasoul e Akram Hospital, Iran University of Medical Sciences, Tehran, Iran.

3. Minimally Invasive Surgery Research Center, Iran University of Medical Sciences, Tehran, Iran.

4. Iran University of Medical Sciences, Tehran, Iran.

5. Scientific Research Center, Tehran University of Medical Sciences, Tehran, Iran.
}

or obese by 2030 (1). In such people, weight loss can improve their social functioning and quality of life $(2,3)$. Treatment options for morbid obesity include low-calorie diet, behavior modification, exercise, and pharmacological and surgical methods (4). Surgical intervention reduces time, removes or improves comorbidities associated with

$\uparrow$ What is "already known" in this topic:

The effect of close supervision on the sport activities after bariatric gastric bypass surgery is not evident.

\section{$\rightarrow$ What this article adds:}

Close supervision was not statistically significant in improving lipid profile and insulin resistance more than the usual telephone follow-up method, but a slightly better effect on BMI was observed, suggesting a cost-benefit trade off assessment in every single bariatric gastric bypass surgery considering specified budgets, patients' health issues, relative urge for weight reduction, etc. 
morbid obesity, and improves quality of life (5).

Roux-en- $Y$ bypass surgery is one of the most common surgical procedures in the treatment of obesity disease (6). It is a popular and effective surgical method for weight loss in morbid obese patients (body mass index [BMI] $\geq$ $35 \mathrm{kgm}^{-2}$ with a comorbid condition that would be improved by weight loss, or BMI $\geq 40 \mathrm{kgm}^{-2}$ ) (7-9). In this procedure, a small stomach pouch is created to restrict food intake, and the connection of the jejunum to the pouch allows food to bypass the duodenum and proximal jejunum.

Sports activities are low cost and noninvasive and have minor side effects (10-12). In addition, regular exercise can reduce risk factors for many diseases, increase energy consumption, accelerate and improve fat oxidation, reduce obesity, especially abdominal obesity $(13,14)$.

There is no doubt about the important role of exercise and diet programs in weight loss and health gain process, however, more studies need to be done to find which kind of exercise planning leads to the best result; and even less is known about whether engaging in exercise after surgery can provide additional improvement in health outcomes (15-17).

However, evidence to support the impact outcomes of postbariatric lifestyle intervention is still limited, but most of the previous studies have shown that postoperative supervised exercise therapy had no significant additional benefit on a short-term weight management outcome and was not able to prevent lean body mass loss; on the other hand, no long-term study has compared the efficacy of supervised or home-based exercise on weight loss and metabolic changes after bariatric surgery (18-21).

Our study aimed at determining which scheduled postoperative exercise therapy, supervised versus home-based exercise, better improves the metabolic biomarkers and reduces insulin resistance and determining whether homebased minimally supervised plans can affect metabolic biomarkers as same as gym-based supervised exercises.

\section{Methods}

\section{Participants}

This randomized controlled trial (ISRCT201111214251N8), which was conducted in Tehran from August 2011 to August 2013, reported a 20-week follow-up result of exercise after gastric bypass surgery.

In this study, 80 obese females, who were candidates for Laparoscopic Roux-en-Y Gastric bypass surgery, were included (after assessment of 108 patients for inclusion and exclusion criteria), using a systemic randomized sampling method from among those receiving medical care in the Research Center for Minimally Invasive Surgery, Rasoul-e-Akram hospital in Tehran, affiliated to Iran University of Medical Sciences.

Entry criteria included age older than 18 years, ability to read and write fluently, and being a candidate for bariatric surgery. Participants were excluded if they had cardiovascular disorders, systolic or diastolic hypertension, uncontrolled metabolic disorders, neuromuscular or rheumatologic disorders, acute pulmonary emboli, chronic symptomatic heart failure, presence of mental or physical disa- bilities, or any postoperative complications.

Participants were randomly (using a random number generator order in an excel worksheet) divided into 2 parallel groups to be evaluated for the effects of exercise in improving insulin resistance and lipid metabolism after bariatric surgery (42 participants underwent home-based exercise therapy and 38 postoperative gym-based exercise therapy) (Figure 1).

All participants were primarily ordered a balanced nutritional regimen based on their BMI and medical condition based on their physicians' decision; the regimen was fully followed by the researcher, while recommending the patients to perform the regimen without any self-changes. Two patients used an anti-lipid medication that was discontinued immediately after the operation according to their surgeon's consultation. Thus, no patient received any anti-lipid medications postoperatively.

\section{Primary Outcome}

The primary outcome for this study was BMI (body mass index). The weight of the patients was measured both at the start and at the end of the study; each time we used a bioelectrical impedance analyzer TANITA ${ }^{\circledR}$ (BC418), the device was recalibrated for each patient. Weight was measured in kilograms with 2 decimals.

Patients' heights were measured in centimeters at the start of the study.

BMI was computed using a SPSS Version 20, with the following formula: weight in kilograms/ height in meteres $^{\wedge} 2$.

\section{Secondary Outcomes}

Lipid Profile and Insulin Resistance: At first, baseline FBS, insulin level, TG, LDL, and HDL were measured using a PARS-AZMOON® lab kit (licensed by ROSH®) and were saved in a SPSS database. After 20 weeks, the same lab measures were repeated at the same laboratory. The measured data were entered into a SPSS database, and homa.ir was calculated using the following formula: fasting serum insulin $(\mathrm{m} \mathrm{IU} / \mathrm{L}) \times$ fasting plasma glucose $(\mathrm{mg} / \mathrm{dl}) /(405)$ as an insulin resistance indicator (25).

Exercise Intervention: For the supervised gym-based group walking, a 5-day a week walk starting from 30 minutes daily and increasing to 60 minutes daily at the end of the first month was planned. They also had 2 gym visits per week at which they started with warm up, biked for 15 minutes without any resistance, then used strength devices according to personal power and strength, and the session ended with another 15 minutes of resistance biking.

For the minimally supervised home-based group walking, a 5-day a week walk starting from 30 minutes daily and increasing to 60 minutes daily at the end of the first month was planned. They had no gym visits, but they were provided with resistance bands and instructed to use them 2 days a week, starting with low intensity bands and progressing to strong. Their activity was followed up with telephone every 2 weeks and in monthly visits.

Participants in both groups received pedometers (Omron brand HJ-321) to monitor intensity and number of their 
steps during their exercise sessions. Therefore, all endurance activities were recorded in this device for each person individually.

At the end of the study, the same factors were studied in the laboratory and BMI was calculated with new measures.

To equalize the groups, all assessments were supervised by 1 senior resident in sports medicine, and the patients were blinded about the number of groups being assessed in this study.

Those postop visit costs, which were related to our study, were paid by Iran University of Medical Sciences, and we caused no extra expenses for our patients.

\section{Statistical Analysis}

The present study reports the follow-up data of 80 postop participants during a 20 -week trial. Because all the follow-up visits and consultations were free, there was no loss to follow-up in this study. Statistical analysis of outcomes was completed using an intention to treat analysis in SPSS Version 20. Significance level was set at $\mathrm{p}<0.05$. Before statistical testing, whenever needed, data were checked for normality. For the factors with normal distribution, mean and standard deviation were provided, and for the factors not following the normal distribution, median and interquartile range were reported. First, baseline data were analyzed for equality in the 2 groups using independent samples t test and Mann-Whitney U test.

At the end, we calculated the amount of change in each factor by subtracting the factor's level at the beginning from the final value; the amount of change between the 2 groups was compared using independent samples $t$ test and Mann-Whitney U test.

\section{Results}

All the participants were female in both groups, and the mean $\pm \mathrm{SD}$ age the participants in the home-based exercise and supervised exercise groups was $38.37 \pm 7.61$ years and $35.67 \pm 6.99$ years, respectively, with no statistically significant difference between the 2 groups (independent $t$ test; $\mathrm{p}=0.126$ ).

The results of the tests and the descriptive statistics of the primary assessments are demonstrated in Table 1 for the factors using $t$ test (mean and standard deviation as descriptive statistics) and in Table 2 for the factors using a nonparametric test (median and interquartile range as descriptive statistics).

Assessing the difference in baseline characteristics between the 2 groups, we found that all the p-values were more than 0.05 . At the end of the study, the same factors were assessed, whose results are displayed in Tables 3 and 4.

\section{CONSORT Diagram}

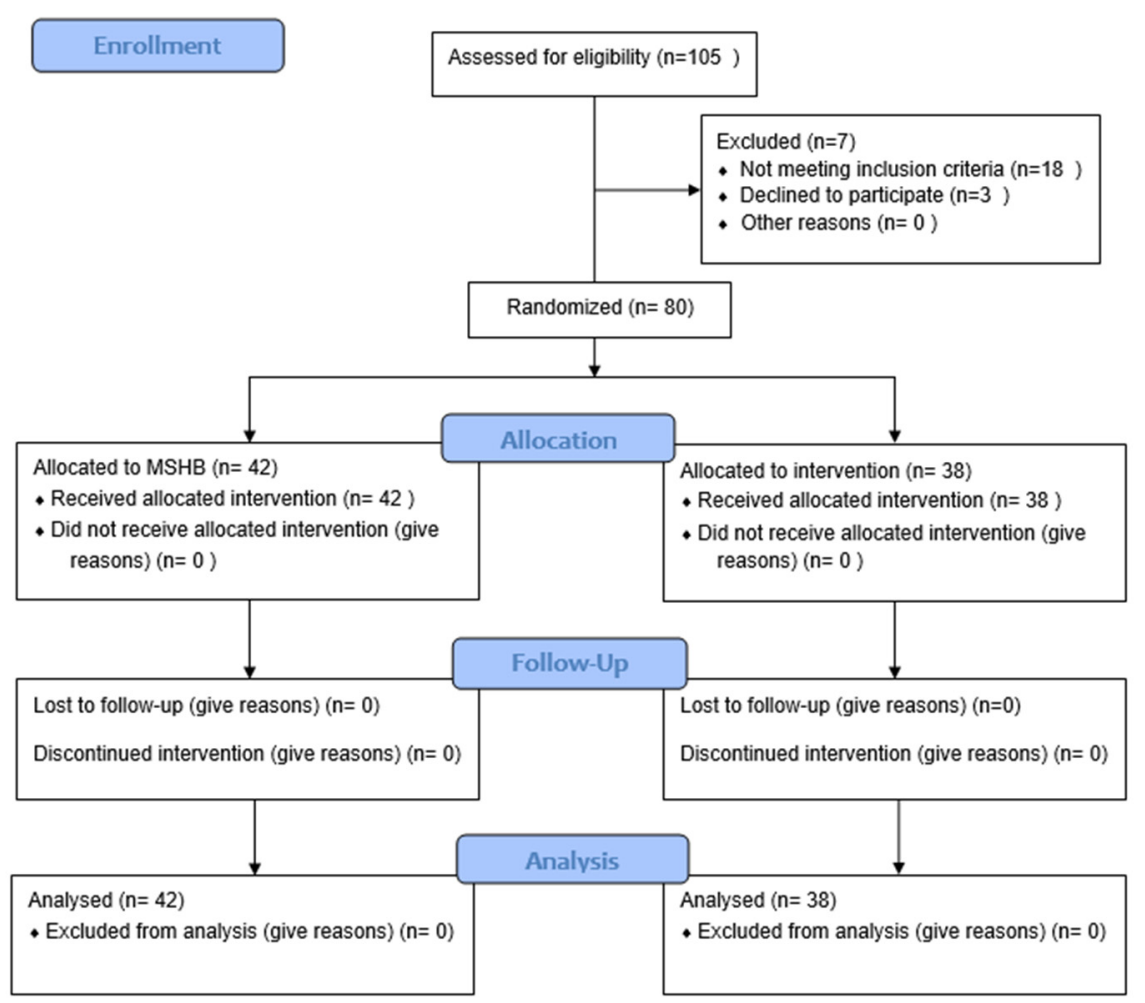

Fig. 1. Consort Flow Chart 
After the study, the changes of the factors (the difference between the quantity of factors before and after the study) were calculated and compared in both groups. The mean change in the amount of BMI, LDL, FBS, TG, HDL, and HOMA.IR was not significantly different between the 2 groups, taking into account the p-values that were all more than 0.05 except for BMI, which had a more significant reduction in CSGB group. The descriptive data for these comparisons are provided in Table 5, and the exact details of the analysis method and test being used are provided in Table 6 (For variables BMI, LDL and FBS an independent samples $t$ test and for other variables, due to lack of assumptions necessary for t test, Mann-Whitney U test was used.).

The mean change (reduction) in BMI was slightly better in CSGB (8.61 95\% CI 7.76-9.45) compared with the MSHB (5.18 95\% CI 3.91-6.46), with $\mathrm{p}<0.01$.

\section{Discussion}

With respect to the effects of exercise on postoperative outcome in patients undergoing bariatric surgery, several studies obtained different results on beneficial effects of different exercise protocols on postoperative weight reduction and physical function; however, a few studies have focused on its beneficial effects on metabolic status and serum metabolic biomarkers $(23,24)$.

Findings of our study mostly indicated that the differences in these methods of exercise are not significant in most aspects. One explanation for similarities in the results between the 2 groups could have been our emphasis on regular biweekly phone contacts with patients in MSHB group and monthly visits. Hence, despite the fact that this group had minimal supervision exercise therapy sessions compared to CSGB group, we still found a slightly significant difference between the 2 groups.

Therefore, we found that using some creative strategies such phone calls, reminder log books, and pedometer could motivate our sedentary patients of MSHB to continue their exercise therapy program; and it is possible that this has positively affected the outcome of exercise treatment in MSHB patients.

The other matter that could be a concern was the cost of postop visits including both direct (the cost of a doctor's visit and health system fees) and indirect costs such as the time lost for the visit and the transportation costs.

During the study not only HbAlc and FBS levels and HOMA-IR index were decreased also TG levels and LDL level and HDL levels were improved. Some studies

Table 1. Baseline data comparison using t-test

\begin{tabular}{|c|c|c|c|c|}
\hline Factor & Group & Mean & SD & Comparison Value ${ }^{*}$ \\
\hline \multirow[t]{2}{*}{ BMI } & $\mathrm{MSHB}^{1}$ & 41.70 & 5.66 & 0.734 \\
\hline & $\mathrm{CSGB}^{2}$ & 42.11 & 5.07 & \\
\hline \multirow[t]{2}{*}{ LDL } & MSHB & 102.73 & 25.47 & 0.691 \\
\hline & CSGB & 100.43 & 25.73 & \\
\hline \multirow[t]{2}{*}{ HDL } & MSHB & 39.71 & 9.84 & 0.397 \\
\hline & CSGB & 41.69 & 10.83 & \\
\hline \multicolumn{5}{|c|}{$\begin{array}{l}\text { * Independent samples } t \text { test; } \mathrm{p} \text {-value of between-group comparison } \\
{ }^{1} \text { Minimally supervised home-based (MSHB) } \\
{ }^{2} \text { Closely supervised gym-based (CSGB) }\end{array}$} \\
\hline \multicolumn{5}{|c|}{ Table 2. Baseline data comparison using Mann-Whitney U test } \\
\hline Factor & Group & Median & Interquartile range & Comparison value \\
\hline \multirow[t]{2}{*}{$\mathrm{TG}$} & MSHB & 128.50 & 91 & 0.725 \\
\hline & CSGB & 122.00 & 51.50 & \\
\hline \multirow[t]{2}{*}{ FBS } & MSHB & 89.50 & 17.25 & 0.908 \\
\hline & CSGB & 91.50 & 16.25 & \\
\hline \multirow[t]{2}{*}{ HBA1C } & MSHB & 5.80 & 1.05 & 0.429 \\
\hline & CSGB & 5.65 & 1.23 & \\
\hline \multirow[t]{2}{*}{ HOMA.IR } & MSHB & 3.00 & 2.16 & 0.732 \\
\hline & CSGB & 2.67 & 1.72 & \\
\hline
\end{tabular}

* Mann-Whitney U Test p-value of between-group comparison

Table 3. Descriptive statistics at the end of the study for variables with normal distribution in both groups

\begin{tabular}{llcc}
\hline Index & Group & Mean & SD \\
\hline BMI & MSHB & 36.52 & 5.51 \\
& CSGB & 33.50 & 4.74 \\
LDL & MSHB & 92.28 & 16.45 \\
& CSGB & 89.48 & 20.43 \\
HDL & MSHB & 44.00 & 8.79 \\
& CSGB & 45.40 & 9.48 \\
\hline
\end{tabular}

Table 4. Descriptive statistics at the end of the study for variables without normal distribution in both groups

\begin{tabular}{lccc}
\hline Index & Group & Median & Interquartile Range \\
\hline Triglyceride & MSHB & 89 & 51.75 \\
& CSGB & 94 & 35.50 \\
FBS & MSHB & 86 & 12.25 \\
& CSGB & 88 & 6 \\
HBA1C & MSHB & 5.30 & 0.90 \\
& CSGB & 5.25 & 0.60 \\
Homa.ir & MSHB & 0.90 & 0.86 \\
& CSGB & 0.93 & 0.70 \\
\hline
\end{tabular}


Table 5. Descriptive statistics for the amount of change in the factors

\begin{tabular}{|c|c|c|c|c|c|}
\hline Variable & Group & Mean & $\mathrm{SD}$ & Median & Interquartile Range \\
\hline \multirow[t]{2}{*}{ Change of BMI } & MSHB & -5.18 & 3.89 & -5.45 & 3.925 \\
\hline & CSGB & -8.61 & 2.76 & -8.46 & 4.175 \\
\hline \multirow[t]{2}{*}{ Change of LDL } & MSHB & -10.43 & 15.60 & 10 & 25 \\
\hline & CSGB & -9.77 & 24.55 & 11.00 & 25.75 \\
\hline \multirow[t]{2}{*}{ Change of HDL } & MSHB & 4.29 & 8.13 & 4.00 & 7.25 \\
\hline & CSGB & 3.71 & 12.56 & 4.00 & 11.00 \\
\hline \multirow{2}{*}{ Change of FBS } & MSHB & -10.18 & 10.92 & -9.5 & 12.5 \\
\hline & CSGB & -6.57 & 12.63 & -3.50 & 18.75 \\
\hline \multirow[t]{2}{*}{ Change of TG } & MSHB & -35.18 & 40.81 & -26.50 & 53.00 \\
\hline & CSGB & -31.84 & 31.90 & -23.00 & 32.75 \\
\hline \multirow[t]{2}{*}{ Change of HOMA.IR } & MSHB & -1.75 & 0.98 & -1.59 & 1.54 \\
\hline & CSGB & -1.78 & 1.05 & -1.66 & 1.35 \\
\hline \multirow[t]{2}{*}{ Change of HBA1C } & MSHB & -0.48 & 9.54 & -0.45 & 0.50 \\
\hline & CSGB & -1.92 & 9.54 & -0.45 & 0.70 \\
\hline Variable & \multicolumn{4}{|c|}{ Statistical Test to Compare the Groups } & $\mathrm{p}$ \\
\hline Change of BMI & \multicolumn{4}{|c|}{ Independent samples t-test } & $<0.001$ \\
\hline Change of LDL & \multicolumn{4}{|c|}{ Independent samples t-test } & 0.890 \\
\hline Change of HDL & \multicolumn{4}{|c|}{ Mann-Whitney U test } & 0.806 \\
\hline Change of FBS & \multicolumn{4}{|c|}{ Independent samples t-test } & 0.177 \\
\hline Change of TG & \multicolumn{4}{|c|}{ Mann-Whitney U test } & 0.881 \\
\hline Change of HOMA.IR & \multicolumn{4}{|c|}{ Mann-Whitney U test } & 0.985 \\
\hline Change of HBA1C & \multicolumn{4}{|c|}{ Mann-Whitney U test } & 0.870 \\
\hline
\end{tabular}

showed that aggressive management improves metabolic outcomes in obese patients $(26,27)$. The data lead to this fact that the combination of surgery and physical activity has been useful to these patients, but the exact effect of each of these methods cannot be inferred from these data, and in this case conducting more studies can be helpful. However, the details and ethical aspects of studies that create groups that do not exercise and have no physical activity guidance remain a problem.

To our knowledge this was the first study to compare the efficacy of minimally supervised home-based and closely supervised gym-based exercise programs on weight reduction and insulin resistance after bariatric surgery, which was the first strength of this study. Second, we used the pedometer that could monitor the intensity and number of steps in the activities, and all endurance activities were recorded in this device for each person individually. In this study, lack of loss to follow-up was achieved by follow-up and incentives that were introduced for our patients, and this was the third strength of our study. On the other hand, ethics and morals did not let us design studies with control groups with no physical activity program, which was the limitation of our study design. However, as a limitation, we did not have any presurgical exercise program (Pre SET) as a lifestyle modification on physical fitness (28). Our second limitation was that most typical gyms do not admit morbid obese individuals because they worry about depreciation of sports equipment, causing limited exercise fields for these individuals. However, we could complete all planned schedules for the supervised group in some gyms, as some special sports instruments could tolerate heavy weights.

\section{Conclusion}

In conclusion, while budget is a matter of concern, the method of postoperative exercise training in obese females undergoing bariatric surgery should not be necessarily under close supervision of a doctor. Moreover, evidence shows that even a remote and creative minimal supervision could be somewhat equally helpful. However, application of this conclusion in medical practice is not yet logical because our study had a sample size of 80 , which is not enough to apply to the medical practice, and thus conducting more focused studies is suggested.

\section{Acknowledgments}

This study was a part of M.D. resident thesis in 2013 supported by Iran University of Medical Sciences. We would like to appreciate all those who participated in this study, the staff of the obesity and sports medicine clinic in Hazrat-e-Rasool hospital. Also, we are thankful to Ms. Pishgah Roudsari for her unsparing assistance.

\section{Financial support}

This study was funded and supported by Iran University of Medical Sciences; Grant No: 90-01-30-13141.

\section{Ethics Statement}

This study was registered with trial registration no. ISRCT201111214251N8. A written informed consent form was obtained from all participants, and the study was approved by the ethical committee of Iran University of Medical Sciences.

\section{Conflict of Interest}

The authors declare that they have no competing interests.

\section{References}

1. Jassil FC, Manning S, Lewis N, Steinmo S, Kingett H, Lough F, et al. Feasibility and impact of a combined supervised exercise and nutritional-behavioral intervention following bariatric surgery: a pilot study. J 
Obes. 2015 Jun 23;2015.

2. McQueen, Matthew A. "Exercise aspects of obesity treatment. Ochs J. 2009;9(3):140-143.

3. Gilmartin J, Bath-Hextall F, Maclean J, Stanton W, Soldin M. Quality of life among adults following bariatric and body contouring surgery: a systematic review. JBI database of systematic reviews and implementation reports. 2016 Nov 1;14(11):240-70.

4. Jiskoot $\mathrm{G}$, Benneheij SH, Beerthuizen A, de Niet JE, de Klerk C, Timman R, et al. A three-component cognitive behavioural lifestyle program for preconceptional weight-loss in women with polycystic ovary syndrome (PCOS): a protocol for a randomized controlled trial. Repd Health. 2017 Mar 6;14(1):34.

5. Braverman-Panza J, Horn DB. Answers to Clinical Questions in the Primary Care Management of People with Obesity: Bariatric Surgery. Fam Pract. 2016 Jul;65(7 Suppl):S23-5.

6. Du X, Zhang SQ, Zhou HX, Li X, Zhang XJ, Zhou ZG, et al. Laparoscopic sleeve gastrectomy versus Roux-en-Y gastric bypass for morbid obesity: a 1: 1 matched cohort study in a Chinese population. Oncotarget. 2016 Oct 8;7(46):76308-15.

7. McQueen MA. Exercise aspects of obesity treatment. The Ochsner Journal. 2009 Sep;9(3):140-3.

8. Wilhelm SM, Young J, Kale-Pradhan PB. Effect of bariatric surgery on hypertension: a meta-analysis. Ann Pharmacother. 2014 Jun;48(6):674-82.

9. Svane MS, Madsbad S. Bariatric surgery-effects on obesity and related co-morbidities. Current Diabet Rev. 2014 May;10(3):208-14.

10. Pratt GM, Learn CA, Hughes GD, Clark BL, Warthen M, Pories W. Demographics and outcomes at American Society for Metabolic and Bariatric Surgery Centers of Excellence. Surg Endosc. 2009 Apr 1;23(4):795.

11. Thorogood A, Mottillo S, Shimony A, Filion KB, Joseph L, Genest $\mathrm{J}$, et al. Isolated aerobic exercise and weight loss: a systematic review and meta-analysis of randomized controlled trials. Am J Med. 2011;124(8):747-755.

12. Chin SH, Kahathuduwa CN, Binks M. Physical activity and obesity: what we know and what we need to know. Obes Rev. 2016 Dec $1 ; 17(12): 1226-44$

13. Caldwell S, Lazo M. Is exercise an effective treatment for NASH? Knowns and unknowns. Ann Hepatol. 2009 Jan 1;8(Suppl 1):S60-6.

14. Coen PM, Tanner CJ, Helbling NL, Dubis GS, Hames KC, Xie H, et al. Clinical trial demonstrates exercise following bariatric surgery improves insulin sensitivity. J Clin Invest. 2015 Jan 2;125(1):248-57.

15. Seale P, Conroe HM, Estall J, Kajimura S, Frontini A, Ishibashi J, et al. Prdm16 determines the thermogenic program of subcutaneous white adipose tissue in mice. JClin Invest. 2011;121(1): 96-105.

16. Timmons JA, Baar K, Davidsen PK, Atherton PJ. Is irisin a human exercise gene? Nature. 2012 Aug 30;488(7413):E9-10.

17. Boström P, Wu J, Jedrychowski MP, Korde A, Ye L, Lo JC, et al. A PGC1-[agr]-dependent myokine that drives brown-fat-like development of white fat and thermogenesis. Nature. 2012 Jan 26; 481(7382):463-8.

18. Coen PM, Tanner CJ, Helbling NL, Dubis GS, Hames KC, Xie H, et al. Clinical trial demonstrates exercise following bariatric surgery improves insulin sensitivity. J Clin Invest. 2015 Jan 2;125(1):248-57.

19. Huck CJ. Effects of supervised resistance training on fitness and functional strength in patients succeeding bariatric surgery. J Stren Cond. 2015 Mar 1;29(3):589-95.

20. Shah M, Snell PG, Rao S, Adams-Huet B, Quittner C, Livingston $\mathrm{EH}$, et al. High-Volume Exercise Program in Obese Bariatric Surgery Patients: A Randomized, Controlled Trial. Obesity. 2011 Sep 1;19(9):1826-34.

21. Stegen S, Derave W, Calders P, Van Laethem C, Pattyn P. Physical fitness in morbidly obese patients: effect of gastric bypass surgery and exercise training. Obes Surg. 2011 Jan 1;21(1):61-70.

22. Beaudoin N, Bouvet GF, Coriati A, Rabasa-Lhoret R, Berthiaume Y. Combined Exercise Training Improves Glycemic Control in Adult With Cystic Fibrosis. Med Sci Sports Exerc. 2016 Sep 23.

23. Dadgostar H, Firouzinezhad S, Ansari M, Younespour S, Mahmoudpour A, Khamseh ME. Supervised group-exercise therapy versus home-based exercise therapy: Their effects on Quality of Life and cardiovascular risk factors in women with type 2 diabetes. Diabetes Metab Syndr. 2016 Jun 30;10(2):S30-6.

24. Herring LY, Stevinson C, Carter P, Biddle SJ, Bowrey DJ, Sutton $\mathrm{CD}$, et al. The effects of supervised exercise training 12-24 months after bariatric surgery on physical function and body composition: A random- ised controlled trial. Int J Obesity. 2017 Jun;41(6):909-916.

25. Keskin M, Kondolot M. Insulin resistance in obese children and adolescents: HOMA-IR cut-off levels in the prepubertal and pubertal periods. J Clin Res Ped Endo. 2010;2(3):100-6.

26. Cadegiani FA, Diniz GC, Alves G. Aggressive clinical approach to obesity improves metabolic and clinical outcomes and can prevent bariatric surgery: a single center experience. BMC Obesity. 2017 Feb 21;4(1):9.

27. Honka H, Koffert J, Hannukainen JC, Tuulari JJ, Karlsson HK, Immonen $\mathrm{H}$, et al. The effects of bariatric surgery on pancreatic lipid metabolism and blood flow. J Clin Endocrinol Metab. 2015 Mar 3;100(5):2015-23.

28. Baillot A, Mampuya WM, Dionne IJ, Comeau E, Méziat-Burdin A, Langlois MF. Impacts of supervised exercise training in addition to interdisciplinary lifestyle management in participants awaiting bariatric surgery: a randomized controlled study. Obes Surg. 2016 Nov 1;26(11):2602-10. 